\title{
Non-brittle rachis 1 (Btr1) gene in genera Triticum L. and Aegilops L.
}

Vavilova V.*, Konopatskaia I., Blinov A., Goncharov N.P.

Institute of Cytology and Genetics, SB RAS, Novosibirsk, Russia

*e-mail:valeriya-vavilova@bionet.nsc.ru

The non-fragility of the spike rachis trait was a key factor in the process of wheat domestication, determined the harvest effectiveness. However, the genetic control of this trait is not well understood in some cultivated and artificial wheat species. It is known that the $Q$ and $T g$ genes are the main regulators of the wheat fragility vs. nonfragility of the spike rachis. Nevertheless, recently it was shown that rachis fragility of the second type (brittle rachis) in diploid wheat is determined by the Btr 1 and Btr 2 genes which are the homologous to those of barley. These genes have been studied only for einkorn wheat species (Triticum monococcum L. and T. boeoticum Boiss.). The study, conducted by Pourkheirandish et al., 2018, was allowed for the conclusion that a single non-synonymous amino acid substitution at 119 position (alanine to threonine) in Btrl gene is responsible for the non-brittle rachis trait in domesticated einkorn wheat. In the present study we investigated variability of $B \operatorname{tr} 1-A$ gene in di-, tetra- and hexaploid wheat species, Btrl-B and Btrl-D genes from Aegilops speltoides Tausch and Aegilops tauschii Coss., respectively. We used a combination of bioinformatical tools and molecular biological methods to determine the full-length sequences of Btrl gene. Btrl-A gene in tetra- and hexaploid wheat species was contained 2 bp deletion at 292 nucleotide position, leading to formation of premature stop-codon.

Acknowledgements: The project was supported by the Russian Science Foundation (grant No. 16-16-10021). 